\title{
Comparative Cost Study for A residential Building Using Different Types of Floor System
}

\author{
Reema R. Nassar ${ }^{1}$, Imad A. Al-Qasem ${ }^{2}$ \\ ${ }^{1}$ Instructor, Department of Civil Engineering, An-Najah National University, Nablus, Palestine. \\ ${ }^{2}$ Lecturer, Department of Civil Engineering, An-Najah National University, Nablus, Palestine. \\ ORCIDs: 0000-0001-8403-4045 (Reema Nassar), 0000-0001-8356-7755 (Qasem)
}

\begin{abstract}
Cost estimation is a key part for any project planning. It's the process of predicting the quantity, cost, and price of the resources required for any project. The differences in construction methods between different forms of slabs result into variation in the cost of the slabs for any building project. Thus, this study aims at assessing the variation in construction cost among various construction methods available for four different types of floor slab systems. A 3D structural model had been analyzed and designed under a statically gravity loads by using computer software (ETABS v.16). In this study; a 3D model had been designed with different slab systems, solid, ribbed (one \& two way), and flat plate slab. After that, quantity survey and cost estimate were done for each system, and a bill of quantity was constructed for each of them. Finally, the most economical system is selected; it is The Flat Plate Slab System.
\end{abstract}

Keywords: Flat plate, Solid slab, Ribbed slab, Cost estimation, Structural Quantities, Bill of Quantity (BOQ).

\section{INTRODUCTION \& RELATED WORK}

Designing and constructing buildings date back to the existence of man on earth, and over the years, various design and construction methods have evolved. These evolutions have led to modern designs and construction methods of various elements of a building; such as floors, wall, ceilings and roofs [1]. Reinforced concrete structures make up of a set of elements that interact together as a one unit to carry the dead loads and live loads placed on the structure safely.

Most reinforced concrete structures are subdivided into slabs and beams, which are subjected primarily to flexure (bending moment) and Shear forces, and columns, which are subjected to axial compression under gravity load and to bending moment and shear force in case of dynamic load. A slab is a structural element and has little thickness comparing to its cross section. [2]

Reinforced concrete slab is a widely used structural element. It provides an economical and versatile method of supporting gravity loads. In addition, the slab also forms integral part of structural frames to resist lateral loads.

These slabs combined with other elements (beams, drop panels or column capitals, etc.) are known as roofing system [3]. There are different types of reinforced concrete slab depending on various criteria such as ribbed slab, flat slab, solid slab, continuous slab, simply supported slab etc.

In general, structural floors/roofs account for the substantial cost of a building in a normal situation. Therefore, any savings achieved in floor/roof considerably reduce the cost of the building [4].

Cost is one of the main constraints of a construction project. Competition in today's construction industry and the importance of cost control are increasing. Accurate estimation of costs in a construction project is one of the major factors for project managers.

Several attempts have been made by researchers to compare the cost among different types of floor systems Zekirija and Isak conducted a comparative study between waffle and solid slab systems. They concluded that the benefits of using a waffle slab system over the solid slab system are significant in the sense of achieving a lighter and economical structure [5]. Besides, Reddy found that the residential building constructed from solid slab requires lesser quantity of material (steel and concrete) by $5.512 \%$ than structure constructed from rib slab [6].

Another study has been developed by MATLAB for optimum design of reinforced concrete slabs (Sahab et al.) [7]. Two types of reinforced concrete slabs, simply supported one-way slab and cantilever slab, have been designed. Cost reduction of $18.92 \%$ and $6.78 \%$ are observed for reinforced cantilever and one-way slab, according to literature.

Elemental cost estimation is one of the methods of approximate estimation which gives a simple and a quick quantification of buildings but it requires significant expertise and judgment in pricing. This method analyzes the cost of the project depending on the element [8].

\section{METHOD}

There are different types of economical floor systems (slabs) for reinforced concrete buildings that nearly satisfy all loading and span conditions. Selecting economical floor systems which can withstand design requirements is discussed. This paper study and examines the elemental cost comparing different types of floor system for residential building in Nablus as a case study. The floor systems used in this study include: solid slab, 
International Journal of Engineering Research and Technology. ISSN 0974-3154, Volume 13, Number 8 (2020), pp. 1983-1991

(C) International Research Publication House. https://dx.doi.org/10.37624/IJERT/13.8.2020.1983-1991

flat plate slab, one-way ribbed slab, and two-way ribbed slabs.

\section{II.I Dataset}

The residential building considered for this study is a typical building similar to a number of buildings constructed in various cities in Palestine.

It is located in Salfit City in Palestine. The building was constructed in 2017 consisting of two floors; ground floor and one top floor with an area equal to $385 \mathrm{~m} 2$, and a staircase with an area equal to $14.3 \mathrm{~m} 2$. Figure 1 shows the columns layout for the residential building.

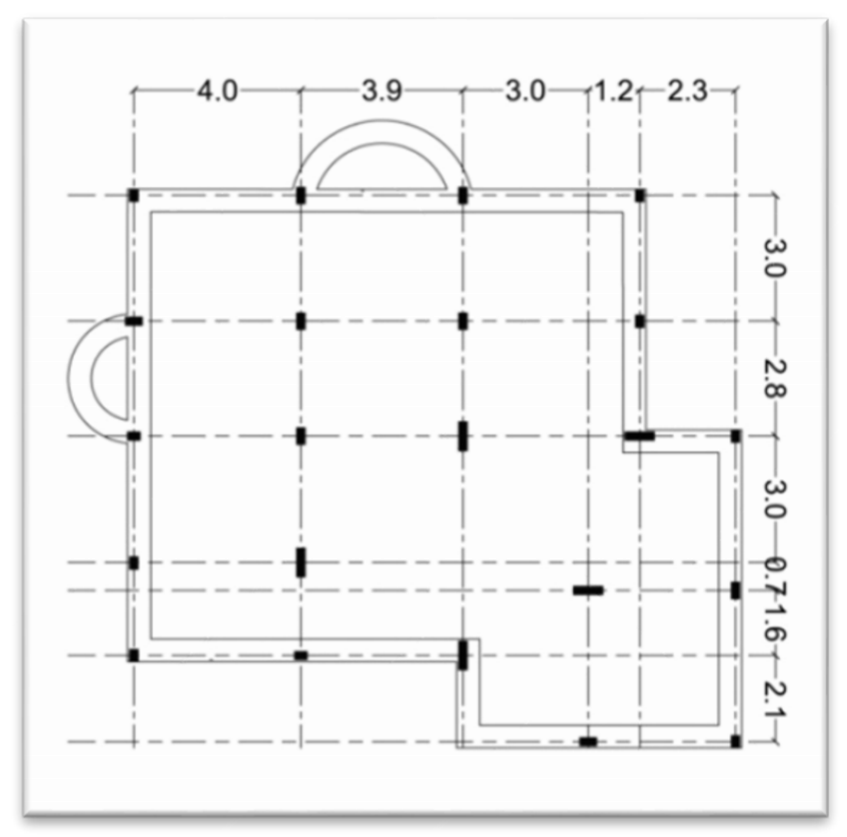

Fig. 1. Columns layout for the building (in meters)

\section{II.II MODELLING, ANALYSIS AND DESIGN ASSUMPTION}

\section{II.II.I Structural Material Properties}

The properties of the concrete and steel used in this research paper shown in table 1

Table 1. Material Properties

\begin{tabular}{|l|l|}
\hline Modulus of Elasticity of concrete, $\mathrm{E}_{\mathrm{c}}$ & $23 \times 10^{3} \mathrm{MPa}$ \\
\hline Compressive strength of concrete, $\mathrm{fc}$ & $24 \mathrm{MPa}$ \\
\hline Modulus of Elasticity of steel, $\mathrm{E}_{\mathrm{s}}$ & $200 \mathrm{GPa}$ \\
\hline Yield Strength & $420 \mathrm{MPa}$ \\
\hline
\end{tabular}

\section{II.II.II Details of the model}

There are four types of floor system considered in this study. These are solid slab (SSS), flat plate (FPS), one-way ribbed slab (OWRS), and two-way ribbed slab (TWRS). The structural elements and types of loading for different types of floor system are shown in table 2

Table 2. Description of different floor system

\begin{tabular}{|c|c|}
\hline Type of structure & Residential building \\
\hline \multicolumn{2}{|l|}{ Flat Plat Slab System } \\
\hline Thickness of slab & $200 \mathrm{~mm}$ \\
\hline Size of edge beams & $\begin{array}{l}300 \mathrm{~mm} \times 200 \mathrm{~mm} \\
450 \mathrm{~mm} \times 200 \mathrm{~mm}\end{array}$ \\
\hline Size of columns & $\begin{array}{l}700 \mathrm{~mm} \times 200 \mathrm{~mm} \\
400 \mathrm{~mm} \times 200 \mathrm{~mm} \\
300 \mathrm{~mm} \times 200 \mathrm{~mm}\end{array}$ \\
\hline Size of footing & $\begin{array}{l}1.4 \mathrm{~m} \times 1.4 \mathrm{~m} \times 0.35 \mathrm{~m} \\
1.6 \mathrm{~m} \times 1.6 \mathrm{~m} \times 0.35 \mathrm{~m} \\
1.85 \mathrm{~m} \times 1.85 \mathrm{~m} \times 0.35 \mathrm{~m}\end{array}$ \\
\hline \multicolumn{2}{|l|}{ Solid Slab System } \\
\hline Thickness of slab & $150 \mathrm{~mm}$ \\
\hline Size of drop beams & $300 \mathrm{~mm} \times 300 \mathrm{~mm}$ \\
\hline Size of columns & $\begin{array}{l}550 \mathrm{~mm} \times 200 \mathrm{~mm} \\
400 \mathrm{~mm} \times 200 \mathrm{~mm} \\
300 \mathrm{~mm} \times 200 \mathrm{~mm}\end{array}$ \\
\hline Size of footing & $\begin{array}{l}1.4 \mathrm{~m} \times 1.4 \mathrm{~m} \times 0.35 \mathrm{~m} \\
1.6 \mathrm{~m} \times 1.6 \mathrm{~m} \times 0.35 \mathrm{~m} \\
1.85 \mathrm{~m} \times 1.85 \mathrm{~m} \times 0.35 \mathrm{~m}\end{array}$ \\
\hline \multicolumn{2}{|c|}{ Two Way Ribbed Slab System } \\
\hline Thickness of slab & $250 \mathrm{~mm}$ \\
\hline Width of the web & $120 \mathrm{~mm}$ \\
\hline Flange Width & $520 \mathrm{~mm}$ \\
\hline Block Dimensions & $40 \mathrm{~cm} \times 25 \mathrm{~cm} \times 17 \mathrm{~cm}$ \\
\hline Size of hidden beams & $\begin{array}{l}450 \mathrm{~mm} \times 250 \mathrm{~mm} \\
300 \mathrm{~mm} \times 250 \mathrm{~mm} \\
\end{array}$ \\
\hline Size of columns & $\begin{array}{l}550 \mathrm{~mm} \times 200 \mathrm{~mm} \\
400 \mathrm{~mm} \times 200 \mathrm{~mm} \\
250 \mathrm{~mm} \times 200 \mathrm{~mm}\end{array}$ \\
\hline Size of footing & $\begin{array}{l}1.2 \mathrm{~m} \times 1.2 \mathrm{~m} \times 0.3 \mathrm{~m} \\
1.5 \mathrm{~m} \times 1.5 \mathrm{~m} \times 0.35 \mathrm{~m} \\
1.8 \mathrm{~m} \times 1.8 \mathrm{~m} \times 0.4 \mathrm{~m}\end{array}$ \\
\hline \multicolumn{2}{|c|}{ One Way Ribbed Slab System } \\
\hline Thickness of slab & $300 \mathrm{~mm}$ \\
\hline Width of the web & $120 \mathrm{~mm}$ \\
\hline Flange Width & $520 \mathrm{~mm}$ \\
\hline Block Dimensions & $40 \mathrm{~cm} \times 25 \mathrm{~cm} \times 17 \mathrm{~cm}$ \\
\hline Size of hidden beams & $\begin{array}{l}700 \mathrm{~mm} \times 300 \mathrm{~mm} \\
400 \mathrm{~mm} \times 300 \mathrm{~mm}\end{array}$ \\
\hline Size of columns & $\begin{array}{l}550 \mathrm{~mm} \times 200 \mathrm{~mm} \\
400 \mathrm{~mm} \times 200 \mathrm{~mm} \\
250 \mathrm{~mm} \times 200 \mathrm{~mm}\end{array}$ \\
\hline Size of footing & $\begin{array}{l}1.3 \mathrm{~m} \times 1.3 \mathrm{~m} \times 0.35 \mathrm{~m} \\
1.55 \mathrm{~m} \times 1.55 \mathrm{~m} \times 0.35 \mathrm{~m} \\
1.75 \mathrm{~m} \times 1.75 \mathrm{~m} \times 0.4 \mathrm{~m}\end{array}$ \\
\hline
\end{tabular}


International Journal of Engineering Research and Technology. ISSN 0974-3154, Volume 13, Number 8 (2020), pp. 1983-1991

(C) International Research Publication House. https://dx.doi.org/10.37624/IJERT/13.8.2020.1983-1991

\section{II.II.III Analysis}

Structural model will be analyzed and designed under a statically gravity load by using computer software (ETABS v.16). The structural elements will be designed as reinforced concrete members according to strength and serviceability criteria as specified in ACI 318-11.

In this study, a 3D model will be analyzed and designed with different slab systems, flat plate, ribbed (one \& two way) and solid slab as shown in figures $2,3,4,5$ respectively. The soil in the site area is mainly rocky where footings will be laid on natural excavated ground, where the bearing capacity of the soil based on the nature of the soil in the site is $280 \mathrm{KN} / \mathrm{m}^{2}$.

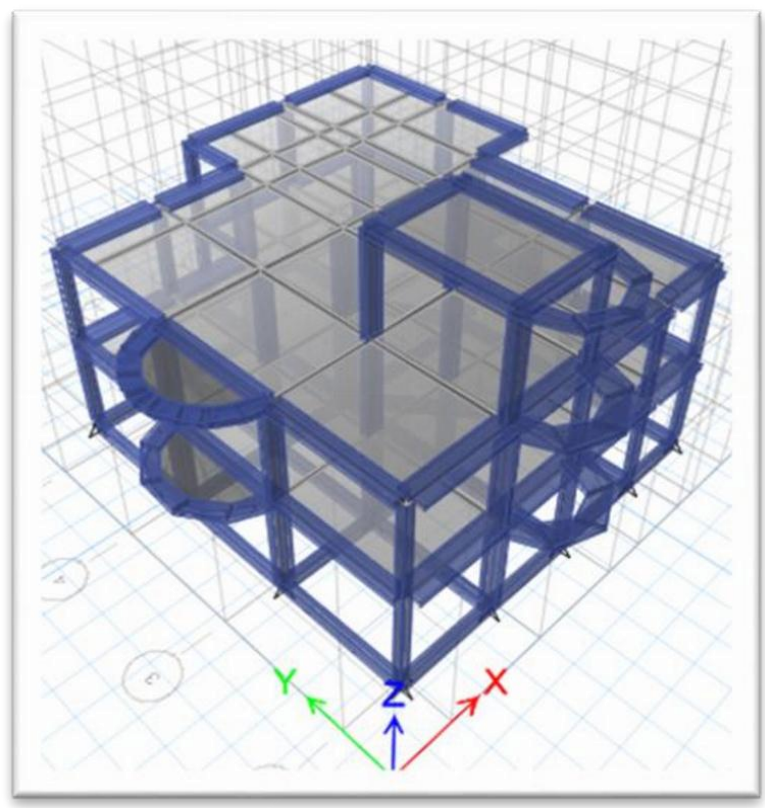

Fig. 2. 3D view for Flat Plat slab model

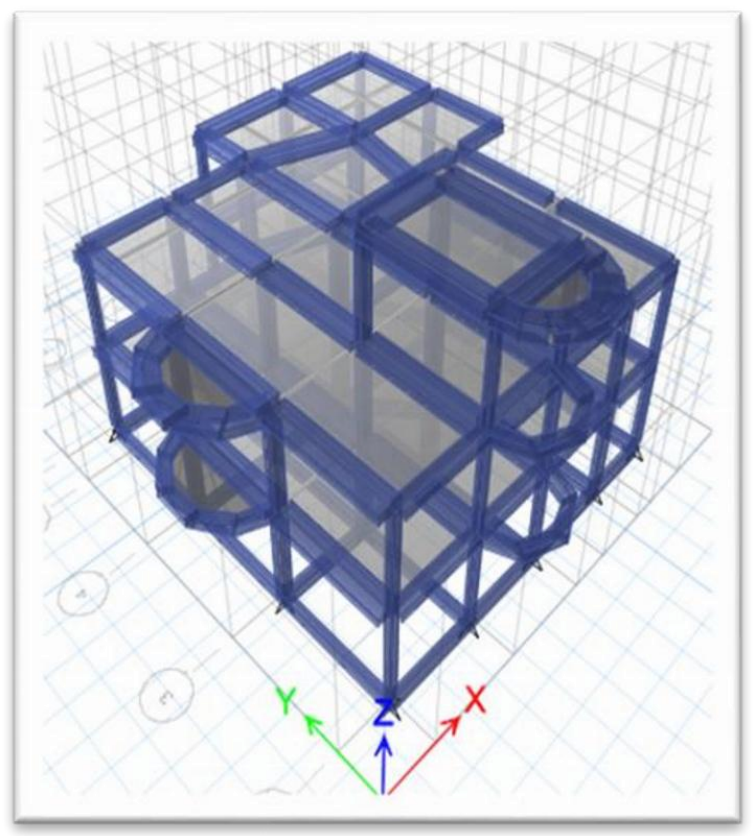

Fig. 3. 3D view for One- Way Ribbed slab model

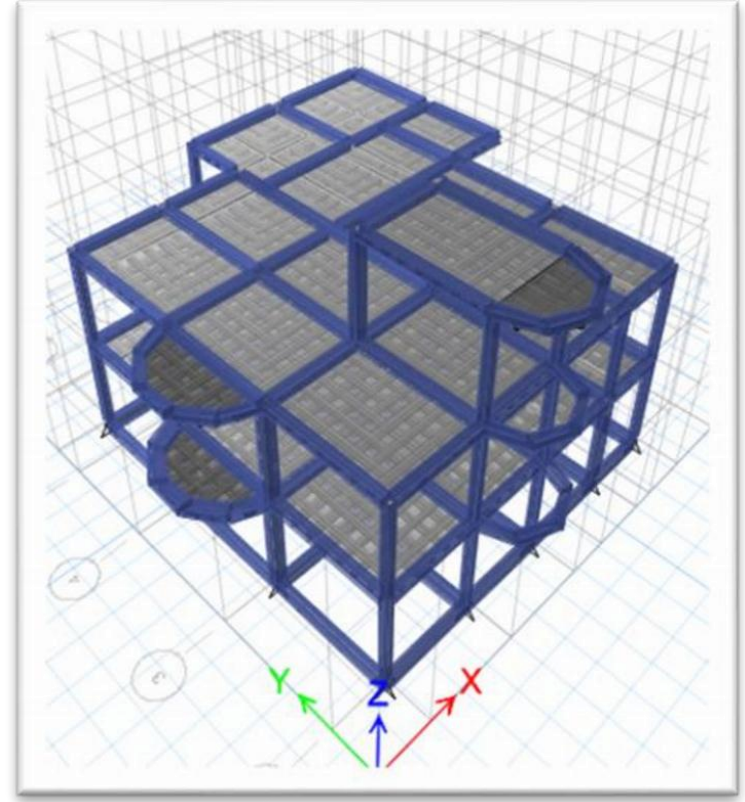

Fig. 4. 3D view for Two Way Ribbed slab model

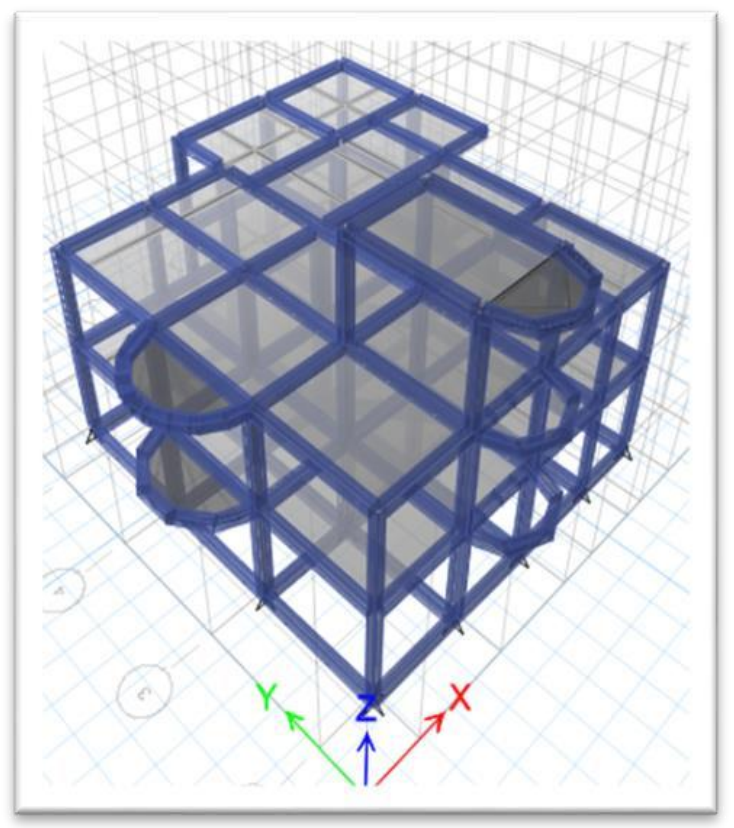

Fig. 5 3D view for Solid slab model

\section{RESULT}

\section{IV.I Structural Quantities}

This part includes quantity surveying work which is required to estimate the quantities of various required materials and the labor involved for satisfactory completion of the construction project. Tables 3 to 6 present the quantities for each element of the building with different floor system. 
International Journal of Engineering Research and Technology. ISSN 0974-3154, Volume 13, Number 8 (2020), pp. 1983-1991

(C) International Research Publication House. https://dx.doi.org/10.37624/IJERT/13.8.2020.1983-1991

Table 3. Quantities estimation of FPS

\begin{tabular}{|c|c|c|c|c|}
\hline $\begin{array}{c}\text { Type of } \\
\text { member }\end{array}$ & $\begin{array}{c}\text { Concrete } \\
\text { Volume }\left(\mathbf{m}^{\mathbf{3}}\right)\end{array}$ & $\begin{array}{c}\text { Steel Bar } \\
(\mathbf{t o n})\end{array}$ & $\begin{array}{c}\text { Formwork } \\
\left(\mathbf{m}^{\mathbf{3}}\right)\end{array}$ & $\begin{array}{c}\text { No. of } \\
\text { Blocks }\end{array}$ \\
\hline Footing & 29.17 & 0.83 & 1.73 & - \\
\hline Column neck & 2.28 & 0.58 & 1.01 & - \\
\hline Columns & 14.42 & 2.48 & 6.55 & - \\
\hline Floor Slab & 81.40 & 6.97 & 20.69 & - \\
\hline Drop beams & 0 & 2.44 & 0 & - \\
\hline Total & $\mathbf{1 2 7 . 2 7}$ & $\mathbf{1 3 . 3}$ & $\mathbf{2 9 . 9 8}$ & \\
\hline
\end{tabular}

Table 4. Quantities estimation of SSS

\begin{tabular}{|c|c|c|c|c|}
\hline $\begin{array}{c}\text { Type of } \\
\text { member }\end{array}$ & $\begin{array}{c}\text { Concrete } \\
\text { Volume }\left(\mathbf{m}^{\mathbf{3}}\right)\end{array}$ & $\begin{array}{c}\text { Steel Bar } \\
(\mathbf{t o n})\end{array}$ & $\begin{array}{c}\text { Formwork } \\
\left(\mathbf{m}^{\mathbf{3}}\right)\end{array}$ & $\begin{array}{c}\text { No. of } \\
\text { Blocks }\end{array}$ \\
\hline Footing & 28.56 & 0.83 & 1.72 & - \\
\hline Column neck & 2.07 & 0.51 & 0.95 & - \\
\hline Columns & 13.11 & 2.18 & 6.15 & - \\
\hline Floor Slab & 61.05 & 6.21 & 20.69 & - \\
\hline Drop beams & 14.06 & 3.63 & 3.64 & - \\
\hline Total & $\mathbf{1 1 8 . 8 5}$ & $\mathbf{1 3 . 3 6}$ & $\mathbf{3 3 . 1 5}$ & - \\
\hline
\end{tabular}

Table 5. Quantities estimation of TWRS

\begin{tabular}{|c|c|c|c|c|}
\hline $\begin{array}{c}\text { Type of } \\
\text { member }\end{array}$ & $\begin{array}{c}\text { Concrete } \\
\text { Volume }\left(\mathbf{m}^{\mathbf{3}}\right)\end{array}$ & $\begin{array}{c}\text { Steel Bar } \\
(\mathbf{t o n})\end{array}$ & $\begin{array}{c}\text { Formwork } \\
\left(\mathbf{m}^{\mathbf{3}}\right)\end{array}$ & $\begin{array}{c}\text { No. of } \\
\text { Blocks }\end{array}$ \\
\hline Footing & 24.7 & 0.75 & 1.53 & - \\
\hline Column neck & 2.06 & 0.42 & 0.99 & - \\
\hline Columns & 12.79 & 2.24 & 5.95 & - \\
\hline Floor Slab & 70.59 & 7.13 & 20.69 & 2236.24 \\
\hline Drop beams & 0 & 4.72 & 0 & - \\
\hline Total & $\mathbf{1 1 0 . 1 4}$ & $\mathbf{1 5 . 2 6}$ & $\mathbf{2 9 . 1 6}$ & \\
\hline
\end{tabular}

Table 6. Quantities estimation of OWRS

\begin{tabular}{|c|c|c|c|c|}
\hline $\begin{array}{c}\text { Type of } \\
\text { member }\end{array}$ & $\begin{array}{c}\text { Concrete } \\
\text { Volume }\left(\mathbf{m}^{\mathbf{3}}\right)\end{array}$ & $\begin{array}{c}\text { Steel Bar } \\
(\mathbf{t o n})\end{array}$ & $\begin{array}{c}\text { Formwork } \\
\left(\mathbf{m}^{\mathbf{3}}\right)\end{array}$ & $\begin{array}{c}\text { No. of } \\
\text { Blocks }\end{array}$ \\
\hline Footing & 27.05 & 0.81 & 1.67 & - \\
\hline Column neck & 2.11 & 0.56 & 0.96 & - \\
\hline Columns & 13.30 & 2.22 & 6.20 & - \\
\hline Floor Slab & 84.77 & 6.42 & 20.69 & 1923.60 \\
\hline Drop beams & 0 & 5.03 & 0 & - \\
\hline Total & $\mathbf{1 2 7 . 2 3}$ & $\mathbf{1 5 . 0 4}$ & $\mathbf{2 9 . 5 2}$ & $\mathbf{1 9 2 3 . 6 0}$ \\
\hline
\end{tabular}

\section{IV.II Cost Estimation}

Cost estimation in construction projects is an important factor for decision making in all the project phases. The cost estimating for construction project starts in the planning phase or in feasibility study to determine the required financial requirements. Then in the construction phase, the actual cost is estimated and compared with the planned cost to assess the variation cost. The successful estimating process essentially depends upon estimator's experience, and acquaintance with achieving an accurate cost assessment; which shouldn't be different a lot from the actual cost.

The results of cost estimation are summarized in table 7 to 10 . For each floor system and regarding the cost of materials and labor of the floor slab system, the results show that the use of flat plate system saves $31.40 \%$ in comparison with solid slab system, $18.27 \%$ in comparison with two way ribbed slab system, and $17.99 \%$ in comparison with one way ribbed slab system.

In addition, the results show that the use of flat plate system compared to other types of slab systems reduces the total cost of construction as shown in figure 6

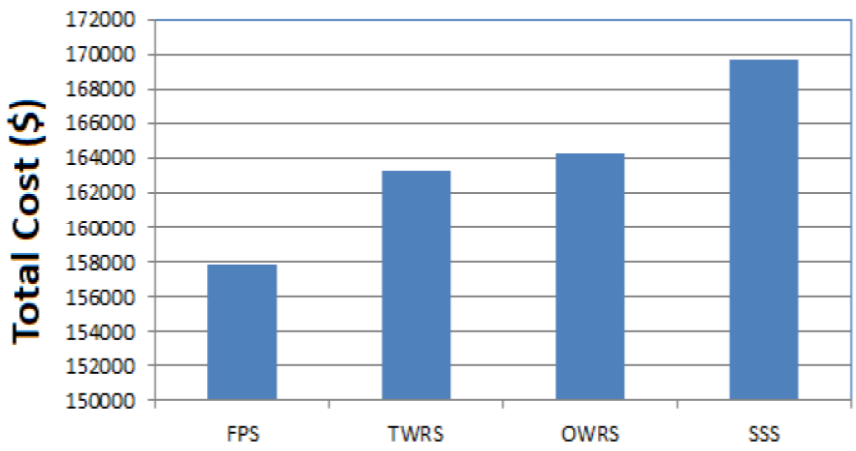

Fig. 6 The total cost of construction for each type 
International Journal of Engineering Research and Technology. ISSN 0974-3154, Volume 13, Number 8 (2020), pp. 1983-1991 (C) International Research Publication House. https://dx.doi.org/10.37624/IJERT/13.8.2020.1983-1991

Table 7. Cost Estimation for Flat Plate System

\begin{tabular}{|c|c|c|c|c|c|c|}
\hline \multirow[b]{2}{*}{ Item } & \multirow[b]{2}{*}{ Description } & \multicolumn{2}{|l|}{ Quantity } & \multicolumn{2}{|c|}{ Unit Price } & \multirow[t]{2}{*}{ Total Price (\$) } \\
\hline & & Value & Unit & Value & Unit & \\
\hline 1 & Excavation for Footing & 329.70 & $\mathrm{CM}$ & 16.04 & $\$ / C M$ & 5288.78 \\
\hline 2 & Footing & 21.66 & $\mathrm{CM}$ & 203.13 & \$/CM & 4399.86 \\
\hline 3 & Column Neck & 2.28 & $\mathrm{CM}$ & 516.31 & $\$ / C M$ & 1177.18 \\
\hline 4 & Tie Beams & 14.06 & $\mathrm{CM}$ & 303.28 & $\$ / C M$ & 4264.16 \\
\hline 5 & Ground Slab & 19.58 & CM & 147.08 & \$/CM & 2879.90 \\
\hline 6 & Columns & 14.42 & $\mathrm{CM}$ & 433.10 & $\$ / C M$ & 6245.31 \\
\hline 7 & Floors Slab & 379.30 & SM & 58.61 & $\$ /$ SM & 22229.82 \\
\hline 8 & Drop beams & 2.44 & Ton & 992.58 & \$/Ton & 2421.89 \\
\hline 9 & Stairs & 6.82 & $\mathrm{CM}$ & 198.49 & $\$ / C M$ & 1353.72 \\
\hline 10 & Sloping Screed & 5.41 & $\mathrm{CM}$ & 102.42 & $\$ / C M$ & 554.11 \\
\hline 11 & Plastering Floors & 1612.96 & SM & 7.14 & $\$ / S M$ & 11516.53 \\
\hline 12 & Painting Floors & 1571.95 & SM & 3.64 & \$/SM & 5724.10 \\
\hline 13 & Tiles & 515.82 & SM & 28.41 & $\$ /$ SM & 14652.02 \\
\hline 14 & Blocks & 113.00 & Box & 105.87 & $\$ /$ Box & 11963.80 \\
\hline 15 & Stone & 693.24 & SM & 60 & \$/SM & 41594.4 \\
\hline 16 & Beam & 6.72 & $\mathrm{CM}$ & 457.08 & $\$ / C M$ & 3071.57 \\
\hline 17 & Doors & 41.75 & SM & 159.66 & $\$ /$ SM & 6665.90 \\
\hline 18 & Windows & 68.02 & SM & 95.20 & \$/SM & 6475.50 \\
\hline 19 & Staircase rail & 15.17 & SM & 47.60 & \$/SM & 722.09 \\
\hline 20 & Staircase Tile & 26.88 & SM & 45.22 & $\$ / S M$ & 1215.51 \\
\hline 21 & Window Stone & 51.44 & SM & 47.60 & $\$ /$ SM & 2448.54 \\
\hline 22 & Granite Stone & 8.00 & SM & 119.00 & $\$ / S M$ & 952.00 \\
\hline & & & Total & & & 157816.7 \\
\hline
\end{tabular}


International Journal of Engineering Research and Technology. ISSN 0974-3154, Volume 13, Number 8 (2020), pp. 1983-1991 (C) International Research Publication House. https://dx.doi.org/10.37624/IJERT/13.8.2020.1983-1991

Table 8. Cost Estimation for Solid Slab System

\begin{tabular}{|c|c|c|c|c|c|c|}
\hline \multirow{2}{*}{ Item } & \multirow{2}{*}{ Description } & \multicolumn{2}{|l|}{ Quantity } & \multicolumn{2}{|c|}{ Unit Price } & \multirow[t]{2}{*}{ Total Price $(\$)$} \\
\hline & & Value & Unit & Value & Unit & \\
\hline 1 & Excavation for Footing & 326.63 & $\mathrm{CM}$ & 15.97 & $\$ / C M$ & 5216.26 \\
\hline 2 & Footing & 21.15 & $\mathrm{CM}$ & 204.32 & $\$ / C M$ & 4322.07 \\
\hline 3 & Column Neck & 2.07 & $\mathrm{CM}$ & 507.50 & $\$ / C M$ & 1052.61 \\
\hline 4 & Tie Beams & 14.06 & $\mathrm{CM}$ & 303.28 & $\$ / C M$ & 4264.16 \\
\hline 5 & Ground Slab & 19.58 & $\mathrm{CM}$ & 147.08 & $\$ / C M$ & 2879.90 \\
\hline 6 & Columns & 13.11 & $\mathrm{CM}$ & 429.20 & $\$ / C M$ & 5628.12 \\
\hline 7 & Floors Slab & 379.30 & $\mathrm{SM}$ & 85.43 & $\$ / \mathrm{SM}$ & 32403.64 \\
\hline 8 & Drop beams & 3.44 & Ton & 1466.71 & \$/Ton & 5040.56 \\
\hline 9 & Stairs & 6.82 & $\mathrm{CM}$ & 198.49 & $\$ / C M$ & 1353.72 \\
\hline 10 & Sloping Screed & 5.41 & $\mathrm{CM}$ & 102.42 & $\$ / C M$ & 554.11 \\
\hline 11 & Plastering Floors & 1612.96 & SM & 7.14 & $\$ / S M$ & 11516.53 \\
\hline 12 & Painting Floors & 1571.95 & $\mathrm{SM}$ & 3.64 & $\$ / \mathrm{SM}$ & 5724.10 \\
\hline 13 & Tiles & 515.82 & $\mathrm{SM}$ & 28.41 & $\$ / \mathrm{SM}$ & 14652.02 \\
\hline 14 & Blocks & 113.00 & Box & 105.87 & $\$ /$ Box & 11963.80 \\
\hline 15 & Stone & 693.24 & $\mathrm{SM}$ & 60 & $\$ / \mathrm{SM}$ & 41594.4 \\
\hline 16 & Beam & 6.72 & $\mathrm{CM}$ & 457.08 & $\$ / C M$ & 3071.57 \\
\hline 17 & Doors & 41.75 & $\mathrm{SM}$ & 159.66 & $\$ / \mathrm{SM}$ & 6665.90 \\
\hline 18 & Windows & 68.02 & $\mathrm{SM}$ & 95.20 & $\$ / \mathrm{SM}$ & 6475.50 \\
\hline 19 & Staircase rail & 15.17 & SM & 47.60 & $\$ / \mathrm{SM}$ & 722.09 \\
\hline 20 & Staircase Tile & 26.88 & $\mathrm{SM}$ & 45.22 & $\$ / \mathrm{SM}$ & 1215.51 \\
\hline 21 & Window Stone & 51.44 & $\mathrm{SM}$ & 47.60 & $\$ / \mathrm{SM}$ & 2448.54 \\
\hline 22 & Granite Stone & 8.00 & $\mathrm{SM}$ & 119.00 & $\$ / \mathrm{SM}$ & 952.00 \\
\hline & & & Total & & & 169717.1 \\
\hline
\end{tabular}


International Journal of Engineering Research and Technology. ISSN 0974-3154, Volume 13, Number 8 (2020), pp. 1983-1991 (C) International Research Publication House. https://dx.doi.org/10.37624/IJERT/13.8.2020.1983-1991

Table 9. Cost Estimation for Two Way Ribbed Slab System

\begin{tabular}{|c|c|c|c|c|c|c|}
\hline \multirow{2}{*}{ Item } & \multirow{2}{*}{ Description } & \multicolumn{2}{|l|}{ Quantity } & \multicolumn{2}{|c|}{ Unit Price } & \multirow[t]{2}{*}{ Total Price (\$) } \\
\hline & & Value & Unit & Value & Unit & \\
\hline 1 & Excavation for Footing & 301.48 & $\mathrm{CM}$ & 16.55 & $\$ / \mathrm{CM}$ & 4989.55 \\
\hline 2 & Footing & 18.10 & $\mathrm{CM}$ & 208.35 & $\$ / \mathrm{CM}$ & 3771.58 \\
\hline 3 & Column Neck & 2.06 & $\mathrm{CM}$ & 468.88 & $\$ / \mathrm{CM}$ & 963.96 \\
\hline 4 & Tie Beams & 14.06 & $\mathrm{CM}$ & 303.66 & $\$ / \mathrm{CM}$ & 4268.40 \\
\hline 5 & Ground Slab & 19.58 & $\mathrm{CM}$ & 147.08 & $\$ / \mathrm{CM}$ & 2879.90 \\
\hline 6 & Columns & 12.79 & $\mathrm{CM}$ & 437.74 & $\$ / \mathrm{CM}$ & 5599.23 \\
\hline 7 & Floors Slab & 379.30 & $\mathrm{SM}$ & 71.71 & $\$ / \mathrm{SM}$ & 27200.98 \\
\hline 8 & Drop beams & 4.72 & Ton & 992.46 & \$/Ton & 4680.27 \\
\hline 9 & Stairs & 6.82 & $\mathrm{CM}$ & 198.49 & $\$ / C M$ & 1353.72 \\
\hline 10 & Sloping Screed & 5.41 & $\mathrm{CM}$ & 102.42 & $\$ / \mathrm{CM}$ & 554.11 \\
\hline 11 & Plastering Floors & 1612.96 & $\mathrm{SM}$ & 7.14 & $\$ / \mathrm{SM}$ & 11516.53 \\
\hline 12 & Painting Floors & 1571.95 & $\mathrm{SM}$ & 3.64 & $\$ / \mathrm{SM}$ & 5724.10 \\
\hline 13 & Tiles & 515.82 & $\mathrm{SM}$ & 28.41 & $\$ / \mathrm{SM}$ & 14652.02 \\
\hline 14 & Blocks & 113.00 & Box & 105.87 & \$/Box & 11963.80 \\
\hline 15 & Stone & 693.24 & $\mathrm{SM}$ & 60 & $\$ / \mathrm{SM}$ & 41594.4 \\
\hline 16 & Beam & 6.72 & $\mathrm{CM}$ & 457.08 & $\$ / \mathrm{CM}$ & 3071.57 \\
\hline 17 & Doors & 41.75 & $\mathrm{SM}$ & 159.66 & $\$ / \mathrm{SM}$ & 6665.90 \\
\hline 18 & Windows & 68.02 & $\mathrm{SM}$ & 95.20 & $\$ / \mathrm{SM}$ & 6475.50 \\
\hline 19 & Staircase rail & 15.17 & $\mathrm{SM}$ & 47.60 & $\$ / \mathrm{SM}$ & 722.09 \\
\hline 20 & Staircase Tile & 26.88 & $\mathrm{SM}$ & 45.22 & $\$ / \mathrm{SM}$ & 1215.51 \\
\hline 21 & Window Stone & 51.44 & $\mathrm{SM}$ & 47.60 & $\$ / \mathrm{SM}$ & 2448.54 \\
\hline 22 & Granite Stone & 8.00 & $\mathrm{SM}$ & 119.00 & $\$ / \mathrm{SM}$ & 952.00 \\
\hline & & & Total & & & 163263.7 \\
\hline
\end{tabular}


International Journal of Engineering Research and Technology. ISSN 0974-3154, Volume 13, Number 8 (2020), pp. 1983-1991 (C) International Research Publication House. https://dx.doi.org/10.37624/IJERT/13.8.2020.1983-1991

Table 10. Cost Estimation for One Way Ribbed Slab System

\begin{tabular}{|c|c|c|c|c|c|c|}
\hline \multirow{2}{*}{ Item } & \multirow{2}{*}{ Description } & \multicolumn{2}{|l|}{ Quantity } & \multicolumn{2}{|c|}{ Unit Price } & \multirow{2}{*}{ Total Price $(\$)$} \\
\hline & & Value & Unit & Value & Unit & \\
\hline 1 & Excavation for Footing & 314.70 & $\mathrm{CM}$ & 16.30 & $\$ / C M$ & 5130.45 \\
\hline 2 & Footing & 20.04 & $\mathrm{CM}$ & 205.58 & $\$ / \mathrm{CM}$ & 4119.39 \\
\hline 3 & Column Neck & 2.11 & $\mathrm{CM}$ & 525.93 & $\$ / C M$ & 1107.21 \\
\hline 4 & Tie Beams & 14.06 & $\mathrm{CM}$ & 303.28 & $\$ / \mathrm{CM}$ & 4264.16 \\
\hline 5 & Ground Slab & 19.58 & $\mathrm{CM}$ & 147.08 & $\$ / C M$ & 2879.90 \\
\hline 6 & Columns & 13.30 & $\mathrm{CM}$ & 429.58 & $\$ / C M$ & 5715.39 \\
\hline 7 & Floors Slab & 379.30 & SM & 71.47 & $\$ / \mathrm{SM}$ & 27109.10 \\
\hline 8 & Drop beams & 5.03 & Ton & 992.46 & \$/Ton & 4990.99 \\
\hline 9 & Stairs & 6.82 & $\mathrm{CM}$ & 198.49 & $\$ / \mathrm{CM}$ & 1353.72 \\
\hline 10 & Sloping Screed & 5.41 & $\mathrm{CM}$ & 102.42 & $\$ / \mathrm{CM}$ & 554.11 \\
\hline 11 & Plastering Floors & 1612.96 & SM & 7.14 & $\$ / \mathrm{SM}$ & 11516.53 \\
\hline 12 & Painting Floors & 1571.95 & SM & 3.64 & $\$ / \mathrm{SM}$ & 5724.10 \\
\hline 13 & Tiles & 515.82 & SM & 28.41 & $\$ / S M$ & 14652.02 \\
\hline 14 & Blocks & 113.00 & Box & 105.87 & \$/Box & 11963.80 \\
\hline 15 & Stone & 693.24 & SM & 60 & $\$ / \mathrm{SM}$ & 41594.4 \\
\hline 16 & Beam & 6.72 & $\mathrm{CM}$ & 457.08 & $\$ / \mathrm{CM}$ & 3071.57 \\
\hline 17 & Doors & 41.75 & SM & 159.66 & $\$ / \mathrm{SM}$ & 6665.90 \\
\hline 18 & Windows & 68.02 & SM & 95.20 & $\$ / \mathrm{SM}$ & 6475.50 \\
\hline 19 & Staircase rail & 15.17 & $\mathrm{SM}$ & 47.60 & $\$ / \mathrm{SM}$ & 722.09 \\
\hline 20 & Staircase Tile & 26.88 & SM & 45.22 & $\$ / \mathrm{SM}$ & 1215.51 \\
\hline 21 & Coping & 51.44 & $\mathrm{SM}$ & 47.60 & $\$ / \mathrm{SM}$ & 2448.54 \\
\hline 22 & Kitchen marble & 8.00 & $\mathrm{SM}$ & 119.00 & $\$ / \mathrm{SM}$ & 952.00 \\
\hline & & & Total C & & & 164226.4 \\
\hline
\end{tabular}




\section{DISCUSSION \& CONCLUSION}

Based on the cost analysis results obtained from this case study, the following conclusions can be drawn:

- $\quad$ For all system floors, the cost constitutes the major part of the total structural cost of reinforced concrete residential building.

- The cost of floor slab may range from 13 to $16 \%$ of the structure cost in building work. The percentages are

- $\quad 13.71 \%$ for FPS, $16.56 \%$ for SSS, $16.23 \%$ for TWSS, and $16.1 \%$ for OWSS of the total cost of structure.

- Using flat plate system is more economical than any other systems.

- The flat plate system is economical since it has no beams. So it can reduce the floor height by (10-15) \%.

- The Results figure out that using flat plate system reduces the total cost of construction by $7 \%$ compared to the solid slab system, $4 \%$ compared to the one way ribbed slab system, and $3.33 \%$ compared to the two way ribbed slab system.

\section{REFERENCES}

[1] S. O. Dosumu and O. A. Adenugo, "Assessment of cost variation in solid and hollow floor construction in Lagos State," Journal of Design and Built Environment, vol. 13, no. $1,2013$.

[2] M. Y. Abdulwahid , I.A. Al-Qasem , I. Arman, "Determination of load transfer in reinforced concrete solid slabs by finite element computer analysis," Journal of Mechanical and Civil Engineering vol. 8 (2013) 1-7. https://doi.org/10.9790/1684-0830107

[3] Kudama L. (2015). Comparison of Equivalent Frame Analysis Results With Finite Element Analysis Results for Flat Plate Slab System, MSc thesis, Addis Ababa University, Addis Ababa Institute of Technology School of Civil and Environmental Engineering.

[4] Ogyiri, V.O. Comparative Cost Analysis of Precast and In Situ Concrete Floor Slabs in Ghana. Master's Thesis, Department of Building Technology, Kwame Nkrumah University of Science and Technology, Kumasi, Ghana,2014

[5] Idrizi Z., and Idrizi I." Comparative Study between Waffle and Solid Slab Systems in Terms of Economy and Seismic Performance of a Typical 14Story RC Building" Journal of Civil Engineering and Architecture 11 (2017) 1068-1076. https://doi.org/10.17265/1934-7359/ 2017.12.002

[6] Halkiyo, J. B., Halkiyu, S. B., \& Reddy, R. R., Dr. (2017). Comparative study of Low Rise Residential Buildings Interms of Plate Stress And Economic Evaluation with Solid Slab and Ribbed Slab: Static Analysis. International Journal of Engineering Sciences \& Research Technology, 6(7), 186-193.

https://doi.org/10.5281/zenodo.823088
[7] Sahab, M.G., Ashour, A.F. and Toropov, V.V. (2005), "Cost optimisation of reinforced concrete flat slab buildings", Eng.Struct., 27(3), 313-322.

[8] Oduro R.,Ankrah A.,Nyako K.,and Tutu E."Cost Analysis of Precast and Cast-in-Place Concrete Construction for Selected Public Buildings in Ghana". Journal of Construction Engineering, Volume 2016, Article ID 8785129, 10 pages http://dx.doi.org/10.1155/2016/8785129 